\title{
La Honorable Hermandad de Cinturones Negros en las artes marciales coreanas
}

\author{
Gonzalo Ariel MILLÁN * (iD) \\ Departamento de Antropología Social, Facultad de Humanidades y Ciencias Sociales, Universidad \\ Nacional de Misiones. Consejo Nacional de Investigaciones Científicas y Técnicas (Argentina).
}

Received: 05/03/2019; Accepted: 08/07/2019; Published: 10/07/2019.

\section{Resumen}

Las artes marciales como el taekwondo y el gumdo constituyen símbolos distintivos de la cultura coreana. Su difusión internacional es el resultado de una política gubernamental sostenida desde mediados del siglo XX. La popularidad de estos sistemas de entrenamiento se asocia a la idea de que inculcan valores fundamentales de la sociedad coreana, como el respeto por la autoridad, la jerarquía y la preeminencia de lo colectivo sobre lo individual. En este trabajo describimos cómo estos valores son incorporados y reproducidos en la práctica cotidiana de artes marciales en Corea, mediante un análisis del sistema de rangos propio de estas disciplinas, y los ritos de paso necesarios para moverse por este espacio simbólicamente estructurado. Al hacerlo, mostramos algunas tensiones y arreglos que surgen de la confrontación entre los valores que encarnan las disciplinas marciales y regímenes morales más amplios. En particular, cómo la enseñanza de artes marciales en Corea contribuye a reproducir relaciones desiguales entre los géneros, perpetuando una forma específica de dominación masculina.

Palabras clave: Artes marciales; deportes de combate; Corea del Sur; ritos de paso; moralidad; dominación masculina.

The Honorable Brotherhood of Black Belts in Korean martial arts

\section{Abstract}

Martial arts such as Taekwondo and Gumdo are distinctive symbols of Korean culture. Its international dissemination is the outcome of a governmental policy sustained since the middle of the 20th century. The popularity of this training systems is associated with the idea that they instill fundamental values of Korean society, such as respect for authority and hierarchy, and the preeminence of collective over individual. In this paper we describe how such values are incorporated and reproduced in the daily practice of martial arts in Korea, by analyzing the rank system typical of these disciplines and the rites of passage needed for moving through this symbolically structured space. In doing so, we show some tensions and arrangements which arise from the confrontation between the values that martial disciplines incarnate and wider moral regimes. Specifically, how the teaching of martial arts in Korea contributes to the reproduction of gender inequalities, preserving a specific form of masculine domination.

Keywords: Martial arts; combat sports; Korea; rites of passage; morality; masculine domination.

\section{A Honorável Irmandade de Cinturões Negros nas artes marciais coreanas}

\section{Resumo}

As artes marciais, tal como o taekwondo e o gumdo, constituem símbolos distintivos da cultura coreana. A sua difusão internacional é o resultado de uma política governamental sustentada desde os meados do século XX. A popularidade destes sistemas de treino se associa à ideia de que inculcam valores fundamentais da sociedade coreana, como o respeito pela autoridade, a hierarquia e a proeminência do coletivo sobre o individual. Neste trabalho descrevemos como estes valores são incorporados e reproduzidos na prática quotidiana das artes marciais na Coreia, mediante a análise dos sistemas de graduações próprios destas disciplinas e os ritos de passagem necessários para se mover neste espaço simbolicamente estruturado. Mostramos algumas tensões e conflitos que surgem na confrontação entre os valores que encarnam estas disciplinas marciais e regimes morais mais amplos. Em particular, como o ensino das artes marciais na Coreia contribui para a reprodução de relações desiguais entre os géneros, perpetuando uma forma específica de dominação masculina.

Palavras-chave: Artes marciais; desportos de combate; Coreia do Sul; ritos de passagem; moralidade; dominação masculina.

\section{Introducción}

Las artes marciales, en especial el taekwondo, son uno de los productos que más han influido en la representación colectiva del pueblo coreano así como en su imagen internacional. En 2010, el ministro de Cultura, Deporte y Turismo anunció que el taekwondo constituye una de las

${ }^{*}$ E-mail: gonzalo.ariel.millan@gmail.com 
imágenes más representativas de la cultura coreana y el "espíritu nacional" (Lee, Gibson \& Yi, 2013). Desde niños, los varones coreanos están expuestos a la influencia de estos sistemas de entrenamiento por medio de la educación pública y privada, el servicio militar obligatorio luego de la mayoría de edad, y los medios de comunicación como el cine, la televisión y las historietas (manwha). Es el resultado de un proceso iniciado tras la independencia del yugo nipón y la consolidación del estado surcoreano posterior a la Guerra de Corea. Este proyecto fue posible gracias a inversiones sostenidas desde el gobierno nacional para la promoción de su principal arte marcial, luego devenido deporte nacional, y el apoyo económico e institucional a las organizaciones marciales que lograran reclamar con éxito su autoctonía. En términos generales, la difusión del fenómeno Hallyu (Ola coreana) contribuyó decisivamente a transformar la imagen internacional del país asiático como productor de automóviles, barcos y tecnología liviana (Nye \& Kim, 2013). En la actualidad, la expansión a escala mundial de las industrias culturales asiáticas desafía la asociación inmediata entre globalización y occidentalización (Forrest \& Forrest-Blincoe, 2018). Las organizaciones de artes marciales coreanas jugaron un papel central en este proceso. Con presencia institucional en más de 180 países, se dedican diariamente a la enseñanza de un sistema de combate típicamente asiático, junto con la historia -al menos una historia- de Corea.

La conexión entre estado nacional y artes marciales en Corea no constituye una novedad. Numerosos trabajos han documentado sus vínculos a través del ejército, el sistema educativo, las actividades deportivas, y más recientemente el turismo y los medios de comunicación (Bowman, 2016; Forrest \& Forrest-Blincoe, 2018; Hwang, 2010; Moenig, 2017; Moenig \& Kim, 2016; Seth, 2008, 2016). En general, hay acuerdo en señalar la eficacia con que el gobierno surcoreano ha construido una representación legítima de las artes marciales como símbolos distintivos de la nación, a partir de disminuir o eliminar de la historia oficial las influencias de Japón y China sobre éstas y, por extensión, sobre otros aspectos de la cultura coreana. El éxito de este proyecto nacionalista es además explicado por una relación de homología entre los valores que inculcan las disciplinas marciales y el ethos cultural de Corea, concebido como colectivista, patriarcal y jerárquico (Lee, Gibson \& Yi, 2013). La principal ventaja de estos estudios radica en que articulan en sus explicaciones procesos económicos, políticos y simbólicos desde una perspectiva histórica. Al hacerlo, sin embargo, suelen aplicar una lectura macro-estructural que presta poca atención a las acciones y experiencias locales que contribuyen a la producción y reproducción cotidiana de estos fenómenos.

El campo de estudios sobre artes marciales y deportes de combate ofrece una alternativa sugerente para comprender fenomenológicamente cómo se internalizan los esquemas cognitivos y emocionales de percepción, apreciación y acción que caracterizan estas disciplinas (Sánchez García, 2008; Sánchez García \& Spencer, 2013; Channon \& Jennings, 2014). Pero aquí corremos el riesgo de caer en el obstáculo inverso. Privilegiar las representaciones de los agentes puede llevarnos a concebir las organizaciones marciales como un cúmulo de historias y experiencias singulares, antes que como un sistema de relaciones y posiciones objetivas en el que se disputan formas específicas de capital cultural y económico. Gran parte de la literatura especializada sobre el tema ha logrado cubrir con éxito ese vacío del que advertía Wacquant en relación a "las diversas maneras en que mundos sociales específicos invisten, dan forma y despliegan cuerpos humanos" $(1999$, p. 238) desde una lógica práctica.

Dando continuidad a este planteo, nuestro trabajo apunta a relacionar esos universos sociales específicos y sus lógicas en el conjunto más amplio de estructuras sociales con las que mantienen relaciones más o menos relativas de correspondencia, de acuerdo al programa para una sociología del deporte inaugurado por Bourdieu en la década de 1980¹. Desde una lectura socioantropológica de algunas disciplinas marciales, intenta arrojar luz sobre la cultura coreana a partir de analizar la práctica marcial como un hecho social total (Mauss, 1979, p. 259), esto es, como un

\footnotetext{
${ }^{1}$ En aquél momento, Bourdieu señalaba que "para comprender un deporte, cualquiera que sea, es necesario reconocer la oposición que ocupa en el espacio de los deportes... Es necesario a continuación poner en relación este espacio de los deportes con el espacio social que en él se expresa. Eso a fin de evitar los errores ligados a la puesta en relación directa entre un deporte y un grupo que sugiere la institución ordinaria... En realidad, la correspondencia, que es una verdadera homología, se establece entre el espacio de las prácticas deportivas... y el espacio de las posesiones sociales" (2007b, p. 173-175).
} 
fenómeno en el que se articulan lógicas correspondientes a un número importante de instituciones y valores en una sociedad dada. Es decir, no como la afirmación vacía de que todo está relacionado con todo, sino como una invitación a buscar en una cultura los simbolizadores nodales comunes a diversas esferas de interacción (Passeron, 2011).

Nuestro análisis de artes marciales como el taekwondo o el gumdo se orienta por la diferenciación entre formas y contenidos propuesta por Simmel (2002) ${ }^{2}$. En este sentido, la característica que distingue a la forma de acción recíproca propia de las artes marciales coreanas consiste en que las relaciones entre practicantes están mediadas por valores como la disciplina, la autoridad, el colectivismo y la desigualdad de género, cristalizados en una estructura institucional jerarquizada a partir de rangos. No obstante, estos valores pueden encontrarse en grados y contenidos muy diversos de la vida social coreana, ya que emergen de una matriz común: la filosofía confucianista y su imbricación en el ethos cultural coreano ${ }^{3}$.

El recorrido que sugerimos empieza entonces por considerar algunos aspectos formales de las organizaciones marciales: el sistema de rangos y los rituales necesarios para moverse dentro de él ${ }^{4}$. El análisis de los rituales más regulares -aunque no menos sacralizados- de las disciplinas marciales nos permite explicar los mecanismos a través de los cuales los agentes fijan el consenso legítimo respecto de determinadas formas de comportamiento y de representación sobre la vida social (Peristiany \& Pitt-Rivers, 2005). A partir de allí, analizamos las implicancias de este sistema para la inculcación y reproducción de repertorios morales de mayor alcance en el sistema cultural coreano.

La predisposición humana a producir valores morales (Howell, 2005), es entendida aquí como "una parte integral del fenómeno, más amplio, de la producción activa de la vida social por parte de sujetos socialmente situados que operan en condiciones relacionales, materiales y simbólicas históricamente engendradas" (Balbi, 2017, p. 14). Éstos poseen una racionalidad propia, que permite a los agentes movilizar repertorios legitimados "para disputar determinados recursos cruciales en y para los colectivos de los cuales forman parte" (Noel, 2014, p. 20-21).

\section{Metodología}

Los datos analizados resultan de una estadía etnográfica de 16 meses en Corea del Sur (abril de 2008 a julio de 2009), y fueron producidos mediante la aplicación de las técnicas de observación

\footnotetext{
2 Procedimiento de abstracción que consiste en extraer "de los fenómenos el elemento de la socialización desprendiéndolo inductiva y psicológicamente de la multiplicidad de sus contenidos y fines..., tal como la gramática separa en el lenguaje las formas puras de los contenidos en los que aquéllas son activas" (2002, p. 50). Este ejercicio analítico está justificado por el hecho de que "en los grupos sociales más diversos en cuanto a sus fines y todo su significado, encontramos las mismas formas de comportamiento de los individuos entre sí" (p. 50). No obstante, la pertinencia epistemológica de este procedimiento no debe llevarnos a creer que tales formas existen con independencia de sus contenidos empíricamente observables (p. 51).

3 Para un estudio socio-histórico sobre la adopción del confucianismo como filosofía estatal y su influencia en la vida cotidiana, ver Hwang (2010), Seth (2008; 2016) y Yang (2008). Un análisis de la estructura y caracterización de la sociedad coreana como colectivista -por contraposición al individualismo occidentalpuede encontrarse en $\mathrm{Na}$ (2008). En relación a la estructura socio-cultural coreana como paternalista, patriarcal y el peso de la jerarquía y la autoridad en las relaciones interpersonales, ver Park (2008). Asimismo, Eun (2008), Lee Jae-kyung (2008) y Lee On-jook (2008) vinculan estos elementos para analizar la estructura familiar coreana, la persistencia de desigualdades de género y la desigual incorporación de mujeres al mercado laboral y a los puestos gubernamentales. Finalmente, a partir de un estudio cualicuantitativo Lee, Gilson \& Yi (2013) muestran el peso que adquieren estos valores en la enseñanza y aprendizaje de taekwondo.

4 Estos elementos son comunes tanto al taekwondo como al gumdo, al taekkyon como al tang soo do, por mencionar las artes marciales coreanas más conocidas. Una excepción importante se da en el caso del arte de la arquería coreana (gungdo), ya que, si bien comparte algunos elementos, como su organización en rangos, por otro lado la interacción entre practicantes, la relación maestro-alumno y la dinámica de las clases, los exámenes y las competencias poseen un estilo bastante diferente. Sería interesante indagar en estas particularidades. No obstante, escapa al objetivo de este trabajo, así como a nuestro conocimiento en profundidad de dicha disciplina. Por ello hemos preferido omitir su referencia.
} 
participante y entrevista en profundidad en academias (dojang) de taekwondo, gumdo (arte coreano de la espada) y gungdo (arte coreano del arco), a las que asistí regularmente durante varias horas por día, cinco días por semana. Incluye también la descripción de mi propio recorrido de aprendiz a iniciado de gumdo bajo la instrucción de Jang (6을 dan y máxima autoridad regional en la disciplina) y Go (5o dan y discípulo principal de Jang), en la isla industrial de Geoje-do, al sureste de la península coreana.

El acceso a las primeras academias de taekwondo fue posible gracias a nuestra participación en un programa de intercambio cultural entre ITF-Argentina e ITF-Korea, en el que se acordó de antemano la inclusión de instructores junior para que dictasen clases en academias de taekwondo pertenecientes a la World Taekwondo Federation (WTF) y de gumdo (World Haidong Gumdo Federation). Esta puerta de ingreso nos permitió ampliar progresivamente la investigación a otras academias de artes marciales, tanto de taekwondo como de gumdo y, en menor medida de gungdo, a partir de la técnica de "bola de nieve", esto es, a través de las relaciones facilitadas por nuestros primeros informantes.

En conjunto realizamos observación participante en diez academias de taekwondo WTF, tres de gumdo y dos de gungdo. Los registros producidos en estas instancias fueron enriquecidos a partir de una visita intensiva de entrenamiento durante una semana a dos academias de taekwondo ITF en Osaka, Japón y la participación en un seminario de dos semanas sobre enseñanza de artes marciales, organizado conjuntamente entre ITF-Argentina e ITF-Korea ${ }^{5}$. Además de las conversaciones informales con maestros, alumnos y compañeros de práctica, realizamos entrevistas en profundidad a un total de quince maestros e instructores de taekwondo y gumdo. Los rangos marciales de los integrantes de la "muestra" fueron los siguientes: 6ㅇ dan (5), 5ㅇ dan (2), 4ㅇ dan (3), 3을 (2), y 2o dan (3). La totalidad de ellos eran varones, debido a que en ninguna de estas academias había mujeres con el rango de cinturón negro. Su edad promedio en 2008-2009 era de 39 años, siendo la edad más baja registrada 27 años y la más alta 53 años.

Un segundo tipo de información que resultó clave para nuestra investigación provino de un corpus documental compuesto por una abundante literatura nativa sobre artes marciales: manuales de entrenamiento, documentos sobre la historia de cada disciplina, biografías y autobiografías de maestros, y revistas de difusión.

\section{Algunas formas nativas de clasificación (marcial)}

En las disciplinas marciales coreanas, el reconocimiento del conocimiento se materializa en símbolos objetivos de jerarquía que contribuyen a organizar las relaciones entre practicantes mediante el establecimiento de códigos que regulan la interacción. La forma ritualizada que adopta la pedagogía de entrenamiento es reforzada por el carácter corporal y pre-reflexivo de transmisión del conocimiento, que permite una comprensión práctica de la actividad (Crossley, 2005; Graham, 2013). El aprendizaje visual y mimético (Wacquant, 2006) de un arte marcial se estructura mediante rituales de elevación de estatus (Turner, 1988), ordenados en una serie escalonada de graduaciones. Su principal función es permitir a los practicantes desarrollar progresivamente las capacidades psicomotrices necesarias para ejecutar las distintas posiciones que componen el corpus técnico de la disciplina. El avance por este sistema de graduaciones supone someterse a evaluaciones periódicas conocidas como exámenes de cinturón, que son supervisadas por un comité evaluador conformado por reconocidas autoridades regionales, y cuya rigurosidad y exigencia física y mental va en aumento.

$\mathrm{Al}$ igual que otras organizaciones jerárquicas, las asociaciones de artes marciales presentan una clara imagen piramidal de distribución poblacional, clasificada por rangos que van desde el cinturón de color blanco hasta el de color negro. Cuando se alcanza el cinturón negro, los rangos se indican mediante finas tiras blancas o doradas cosidas a la punta del cinturón, denominadas dan. Estos distintivos forman parte de la indumentaria obligatoria para la práctica cotidiana y son el

${ }^{5}$ El mismo tuvo lugar en la ciudad de Busan durante el mes de enero de 2009, y contó con la participación de

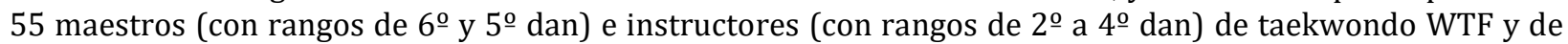
gumdo provenientes de distintas ciudades y pueblos de la región. 
principal símbolo de reconocimiento de las jerarquías en los gimnasios. La graduación es reforzada por una gestualidad que promete distinción mediante la aplicación de estrictas reglas de etiqueta, como las reverencias iniciales y finales hacia la bandera nacional, institucional y al maestro, los saludos entre practicantes, la organización del grupo en las distintas etapas del entrenamiento, la secuencia en que son transmitidas las ordenes, la regulación de los tiempos en que se divide la sesión, entre otras.

Esta compleja rutina ceremonial hace "manifiesto el estatus honorable de los actores" (Peristiany \& Pitt-Rivers, 2005, p. 2, traducción propia), y su repetición produce un compromiso dóxico con las formas instituidas de autoridad. La eficacia simbólica que otorga legitimidad a la autoridad conferida por las artes marciales radica en el reconocimiento social de la institución que reconoce. Su aprobación colectiva, es decir, objetiva, es activada regularmente en cada nuevo acto público de institución -como los exámenes anuales de cinturones-, pero también en las deferencias más casuales e igualmente importantes donde se nombra a alguien por su rango (Maestro, Instructor, Profesor, etc.). Otorgando así una "muestra de reconocimiento con respecto a la persona en particular a la que se dirigen, pero sobre todo con respecto a la institución que ha instituido a ésta" (Bourdieu, 1993, p. 122), y que constituyen otras tantas formas cotidianas de actualizar la tradición confuciana de reverencia a la figura del maestro/profesor.

Entre las instancias de evaluación que un practicante debe sortear durante su carrera como artista marcial, el examen para obtener el primer dan de cinturón negro es la más significativa para la mayoría de los practicantes. Por un lado, la mayor tasa de deserción en las academias analizadas se registra luego de que el alumno obtiene el cinturón negro, esto es, aproximadamente un año después de iniciado su entrenamiento. Estadísticamente hablando, este examen es la prueba más exigente y la última que experimentan la gran mayoría de los practicantes.

Desde un punto de vista subjetivo, en cambio, la diferencia entre cinturones de color y cinturones negros tiene un valor agregado. Habilita a nuevas instancias de interacción social antes vedadas, debido al estatus social que otorga al iniciado. En este sentido, el cinturón negro no es algo que se posee sino algo que se es, a fuerza de una reinscripción de la experiencia corporal en el orden simbólico (Bourdieu, 2007a). En particular, mediante el desplazamiento del cuerpo por el espacio simbólicamente estructurado de las jerarquías marciales. Más aún, la costosa elección de inscribir a un hijo en una academia de artes marciales resulta una apuesta a largo plazo que muchas madres -las principales encargadas de planificar el presupuesto familiar, seleccionar las escuelas y las actividades extra-curriculares en las familias surcoreanas- consideran razonable (Millán, 2015). La obtención de este capital cultural posicionará a sus hijos en una situación de ventaja relativa frente a aquellos que no lo poseen. Por ejemplo, durante el servicio militar obligatorio para varones (Moenig, 2017), permitiéndoles faltar a varias sesiones de entrenamiento semanal. Además, les proveerá de una rápida salida laboral como instructores ayudantes en alguna de las miles de academias privadas de artes marciales -una opción tan desgastante como mal remunerada, según los estándares nacionales-, que les garantizará un ingreso regular durante sus estudios superiores, hasta que consigan insertarse en una mejor ocupación, o decidan hacer carrera en una disciplina marcial, ya sea estableciendo su propia academia o a través de la competencia deportiva. En suma, aprender un arte marcial constituye una estrategia en la que se articulan una serie de variables que no se acotan en la valoración sobre las posibles ventajas que produce la práctica marcial o deportiva en términos de formación de la persona, como suele argumentarse desde el ámbito de la salud o de la psicología (Graham, 2013), aun cuando a éstas últimas se le pueda asignar un rol importante.

\section{Ser un cinturón negro en la cultura coreana}

La transformación en cinturón negro implica un acto de investidura simbólica en el que se celebra un tipo particular de virtud humana. El lenguaje asiste a este proceso en una forma que no se limita a la comunicación, sino que "tiene un valor ontológico, moldea de nuevo el ser mismo del neófito" (Turner, 1988, p. 110). El alumno que aprueba el examen de primer dan no sólo obtiene un cinturón negro, se convierte en uno. "Soy primer dan" (Il dan ieyo), la fórmula repetida con frecuencia para informar sobre la graduación, designa, además de una posición en la jerarquía marcial, un estado del ser en el mundo. 
De quien es cinturón negro se espera que exprese en carne viva la disciplina y los valores morales que promulga el arte marcial, como representante y garante de la reputación social de la academia. Al instituir una diferencia entre practicantes -y entre personas- la institución reconoce una competencia sobre la que pesa un imperativo moral; "es un derecho de ser que es un deber ser (o de ser)" (Bourdieu, 1993, p. 115). Responsabilidad que los practicantes experimentan con considerable angustia, como lo indican los comentarios acerca de no sentirse preparado para ser cinturón negro, que surgen a menudo en las conversaciones informales antes y después de las sesiones de entrenamiento cercanas a la fecha de examen. Detrás de este sentimiento, asoma la convicción de que cumplir con esta responsabilidad conlleva una pesada carga emocional.

El inicio de la fase liminal se caracteriza por una suspensión momentánea de algunas formas de interacción que en circunstancias normales orientan las rutinas de entrenamiento. En las últimas semanas de preparación, las normas de etiqueta se relajan. El maestro y sus ayudantes permiten a los que van a ascender de estatus experimentar marginalmente la sensación de pertenecer al rango superior. Es normal en esta etapa que se obvien algunos comportamientos típicos de subordinación, como el uso de sufijos honoríficos durante las conversaciones informales ${ }^{6}$. En una cultura donde la distancia etaria y social se expresa mediante un elaborado lenguaje que implica el manejo preciso de sufijos de respeto, y cuyo uso es celosamente controlado por los interlocutores, resulta notable la manera en que se descuida o tolera su infracción en las semanas previas al examen. Incluso se permite a los que atraviesan esta etapa participar junto al maestro y su séquito de instructores de actividades sociales fuera del gym, invitándoles a beber juntos en los luminosos bares de neón que rodean los astilleros donde trabaja la mayor parte de la población masculina de la isla, pagándoles los tragos y la comida.

Contrariamente, a medida que se acerca la fecha crucial los entrenamientos diarios dentro del ámbito del dojang reflejan la suspensión simbólica de "las categorías y grupos en el orden social estructurado [...] los neófitos son meros entes en estado de transición y, por el momento, sin lugar ni posición" (Turner, 1988, p. 109). Entre cuatro y seis semanas antes del examen, los aspirantes son arrojados fuera de la temporalidad grupal que otorga el distintivo carácter de comunión a la práctica marcial. Mientras la clase continúa su ritmo normal, luego del saludo inicial los alumnos en situación liminal son apartados del grupo, a un costado y atrás, regulando su propio tiempo, conjuntamente y a destiempo del resto, aunque siempre bajo la mirada atenta del maestro. Únicamente los saludos más formales al inicio y al final de cada entrenamiento los designan como miembros del colectivo mayor, que de esta manera ya no actúa como espejo para los aspirantes. Ahora deben autocorregirse. Se les exhorta a compartir más tiempos entre ellos y prestarse atención mutuamente, ensayando exclusivamente aquellos aspectos técnicos que van a ser evaluados. Estas acciones contribuyen a "desarrollar entre los neófitos una intensa camaradería e igualitarismo" (Turner, 1998, p. 102).

A medida que se acerca la fecha, la experiencia de liminalidad se acentúa y la distancia de apenas un rango que separa al cinturón de color del dan parece ensancharse. A los ojos de los aspirantes, los movimientos de los últimos parecen dotados de una destreza, agilidad y autocontrol corporal inalcanzables, que alimentan la percepción de que sus portadores poseen cualidades humanas superiores, "como si estuvieran dotados de un tipo de carisma grupal, de una virtud específica que comparten todos sus miembros y de la que carecen los demás" (Elias, 2016, p. 28).

El maestro del dojang, "director de orquesta" que conoce el clima general de su gimnasio, se asegura de alimentar esta percepción con arengas sobre la distinción de pertenecer a la

\footnotetext{
${ }^{6}$ El idioma coreano -hangul- tiene una estructura gramatical con una fuerte impronta confuciana, a partir de la cual el estatus y la distancia social entre los interlocutores se expresa por medio de sufijos honoríficos de cortesía. Su uso sirve tanto para referirse a personas como para conjugar verbos. Existen siete niveles que designan la "distancia" social -ya sea ésta de edad, parentesco, laboral, educativa, etc.- entre hablante y oyente. Los primeros seis se conocen como jondaemal o lengua honorífica, mientras que el nivel más bajo se denomina banmal. A cada uno de estos niveles corresponde un sufijo, que se deriva de la forma imperativa no honorífica del verbo. En este sentido, a mayor grado de familiaridad, más relajada se vuelve la necesidad de recurrir a un sufijo "elevado", en especial aquellos que indican distancia etaria entre interlocutores. Asimismo, el entorno social ejerce un fuerte control sobre el uso de los sufijos honoríficos, particularmente en situaciones formales de interacción, tales como las que ocurren en el ámbito de entrenamiento marcial.
} 
"hermandad de los cinturones negros" (geom-eun saeg belteuui hyeongjeae)7, aludiendo al estatus que confiere la graduación, el imperativo de comportarse de acuerdo a los principios de virtud y de generosidad, de respetar a los mayores y llevar una vida honesta, disciplinada y responsable. Estas acciones contribuyen a que los aspirantes y los demás cinturones negros se sientan partícipes de una comunidad que une a los practicantes de la disciplina a lo largo y ancho del planeta, diluyendo, al menos idealmente, diferencias étnicas, religiosas, de género y clase social, en un acto mágico de hermanamiento similar a los grupos de edad descritos por los antropólogos (e.g., Evans-Pritchard, 1992). Mediante un lazo simbólico de sangre -fundado sobre ideales como el sacrificio compartido, la tolerancia al dolor, la disciplina y la constancia-, los alumnos que atraviesan juntos la ceremonia de graduación establecen un vínculo de "parentesco ficticio" (Wacquant, 2012), que los habilita a utilizar los apelativos de hermano mayor (hyeong) y hermano menor (dongsaeng). Además, la ceremonia instituye en ellos lazos de familiaridad temporal con otros practicantes -los que participaron previamente del rito y los que previsiblemente participarán- sin disolver las jerarquías internas, marcando una diferencia con la communitas descrita por Turner (1988).

Desconocer el efecto mágico ${ }^{8}$ de este rito de institución supone negar uno de los aspectos más contradictorios de la moralidad implícita en las artes marciales coreanas: que establece una frontera de género contraria a la declaración discursiva de las organizaciones marciales sobre su acceso igualitario para ambos sexos. La efectividad simbólica de este acto social descansa en el hecho de que parte de diferencias objetivas, como las diferencias biológicas entre sexos, a las que se le sobreimprimen cualidades sociales, tales como aquéllas que en la cultura coreana asocian lo femenino a la fragilidad y la docilidad (Kim, 2012), características que contrastan con la disciplina, el autocontrol y la fortaleza asociada a las artes marciales. Desconocer este efecto social, además, supone negar el mundo cultural más amplio en el cuál las artes marciales coreanas se desarrollan, y cuyas estructuras constriñen las acciones y trayectorias de los practicantes, no ya como artistas marciales sino como agentes en una sociedad determinada.

\footnotetext{
${ }^{7}$ Este término no es de uso oficial, ni tampoco tan corriente como nuestro relato puede sugerir. La referencia histórica más antigua que encontramos remite a 1959, durante la primera gira internacional para presentar el taekwondo, dirigida por el Gral. Choi. En aquella ocasión, el equipo militar realizó una visita a Vietnam del Sur y Taiwán en la que Choi formuló tres ideales básicos para los practicantes. El segundo de los cuales afirma, "Debemos unirnos con todos los hombres en una hermandad de sangre sin importarnos la religión, raza, nacionalidad o fronteras ideológicas" (Choi, 1996, p. 24). En 1965, cuando escribe la famosa enciclopedia del taekwondo, señala que con la obtención del $1^{\mathfrak{0}}$ dan, "el cinturón negro también entra a una nueva responsabilidad. Aunque es joven, ha entrado en la fraternidad honorable de los cinturones negros del mundo, y sus actos en el exterior como en el interior de la sala de entrenamiento serán minuciosamente observados. Su comportamiento se refleja sobre todos los cinturones negros, debiendo esforzarse constantemente en dar el ejemplo a las categorías inferiores" (1996, p. 727. Nuestro énfasis). En las semanas previas al examen para cinturón negro de taekwondo o de gumdo, los maestros suelen hacerse eco de este término cuando hablan con los aspirantes sobre las responsabilidades que deberán asumir si logran aprobar el examen. Aunque no hemos podido establecer claras referencias bibliográficas para estas disciplinas, su uso compartido es indicativo de que proviene de una matriz común.

8 Según Bourdieu, la eficacia simbólica de los ritos de institución, como los exámenes de cinturones en las artes marciales, descansa en "el poder que poseen de actuar sobre lo real actuando sobre la representación de lo real" (1993, p. 4). En este sentido, "La institución es un acto de magia social que puede crear la diferencia ex nihilo, o bien, y éste es el caso más frecuente, explotar de alguna forma unas diferencias preexistentes, como las diferencias biológicas entre los sexos...” (1993, p. 4). El carácter mágico que Bourdieu asigna a los actos de institución tiene su base, según lo entendemos, en el análisis weberiano de la racionalidad. Para Weber, los tipos de acción social se ordenan según una escala decreciente de racionalidad (2014, p. 99) en la que la magia, al igual que la ética religiosa, se ubican más próximas al polo irracional. Es necesario aclarar que el argumento de Weber no tiene en este aspecto una carga moral -de hecho, uno de los principios epistemológicos de la sociología comprensiva es la irracionalidad moral del mundo-, sino que se asocia a la posibilidad de articular con un mayor o menor grado de conciencia los distintos elementos -medios y finescon que los actores otorgan sentido a sus acciones. La frontera simbólica que las artes marciales coreanas imponen a las mujeres, entonces, se asocia al hecho de que los actores no son plenamente conscientes de que están operando en esta dirección. Este aspecto contrasta de lleno con el carácter racional, sistemático y codificado que caracteriza la enseñanza de las disciplinas marciales en la actualidad.
} 
Los dos relatos que siguen nos ayudarán a comprender el sentido que las apuestas simbólicas y materiales implicadas en la adquisición de un rango marcial tienen para el grupo de practicantes y para otras personas de la sociedad coreana. El primero, relativo al ritual para la obtención del cinturón negro de gumdo. El segundo, sobre un conflicto por una transgresión patrimonial y su resolución mediante la apelación al prestigio social del artista marcial. La referencia exclusiva al gumdo no debe hacernos perder de vista que estos elementos, entendidos como forma de acción recíproca, son comunes a otras disciplinas marciales coreanas ${ }^{9}$.

\section{La iniciación, un momento sagrado}

a) "Hoy es el gran día. Las últimas semanas han sido muy intensas, tanto física como mentalmente. Los últimos cinco días fueron particularmente exigentes, con tres y hasta cuatro horas de entrenamiento diario bajo la estricta supervisión de Jang. Nuestro punto de encuentro es su gimnasio, que está rebosante por la llegada de alumnos de las academias de Minho, su hermano mayor, y de Go, su principal discípulo. Desde allí partiremos en tres vehículos hacia la ciudad de Kimhae, donde tiene lugar dos veces por año el examen para cinturones negros. Casi todos vamos a rendir el primer dan, excepto un estudiante que rinde para segundo dan y dos veteranos de mi edad que darán la dificilísima prueba para cuarto dan. El grupo de Jang está compuesto por tres adultos, dos adolescentes y siete niños de entre diez y once años.

Entrando a la ciudad de Kimhae empezamos a avistar camionetas con las insignias distintivas de nuestra asociación. El estacionamiento del estadio está repleto y nos toma un tiempo encontrar lugar para estacionar. Los chicos corren en bandadas con sus dobok negros y sus espadas de madera (moggeom) sujetadas a los cinturones. Los instructores, vistiendo camisas rosado claro, corbatas rozado oscuro y pantalones de vestir negro -uniforme oficial para exámenes-, intercambian saludos apresurados mientras tratan de controlar a sus pupilos. Hay grupos de instructores revoloteando alrededor de maestros que usan trajes elegantes color gris; son los directores de área, máximas autoridades de gumdo a nivel provincial. El estadio tiene unos setenta metros de largo. Al frente, las principales autoridades se acomodan en sus asientos, sobre una plataforma de madera erigida a metro y medio de altura.

La Asociación Coreana de Haidong Gumdo se divide en nueve áreas, una por cada provincia y una por Seúl. Cada zona es presidida por un maestro y varios directores y sub-directores. Nuestra provincia, Gyeongsang-do, se divide en cinco áreas y nueve sub-áreas, pero únicamente los directores y el presidente de la zona pueden sentarse en la tarima. Casi todos los instructores y maestros son a su vez evaluadores. Cuando llegamos, parecen ocupados en organizar las filas, cubrir con manteles las mesas, preparar los soportes para los cortes y alinear a los 150 estudiantes que participamos de la ceremonia ese día. En las tribunas laterales, familiares y amigos se acomodan en las gradas.

Todavía con las zapatillas puestas nos llaman a formar. Apurados, nos ayudamos unos a otros para atar las cintas numeradas que nos dieron al inscribirnos. Un maestro oficia de locutor al costado de la tarima. La voz a través del micrófono suena mecánica y tapa el murmullo general producido por alumnos, familiares y maestros. Empieza a sonar el himno nacional y el estadio se sume en un silencio reverente. A continuación, saludamos a los directores diciendo ¡haidong!, acompañado de una acentuada caravana con el sable al frente y la punta en dirección al piso. El locutor retoma su lugar y empieza a presentar uno por uno a los maestros principales, de acuerdo a su nombre y rango. Cada presentación es seguida de un saludo colectivo y aplausos desde las tribunas. Cuando termina la presentación, giramos sobre nuestros talones hacia la derecha y saludamos colectivamente a los demás instructores situados en fila al costado del estadio. Para cerrar la ceremonia inaugural, se lee un breve discurso sobre el deber del cinturón negro para con sus maestros, familia y la sociedad en general, y nos mandan a sentar al fondo del recinto.

El examen se divide en tres partes: formas, prueba física y corte. Estas secciones varían notablemente entre un tipo de examen inicial, como el de primer dan, y otros más elevados, así como de acuerdo a la edad del aspirante. En estas ceremonias, al igual que en los torneos, se hacen

${ }_{9}^{9}$ Para un análisis similar que toma al taekwondo WTF como referencia etnográfica, ver Millán (2015). 
particularmente legibles las diferencias de estilo -más deportivo o tradicional- entre las escuelas de cada maestro.

La mayoría de los alumnos tienen entre once y trece años. Nos llaman en oleadas de cinco en cinco, mientras el resto prepara el cuerpo con ejercicios de calentamiento físico dirigidos por un maestro. En esta instancia se evalúan aspirantes a cinturón negro desde el primer dan hasta el cuarto dan. Los exámenes de rangos inferiores son tomados por los maestros de cada dojang. A partir del cuarto dan, los practicantes de todo el país se reúnen una vez por año en la ciudad de Gwanju, sede de la Federación Mundial de Haidong Gumdo y donde reside su presidente y fundador, que en la actualidad es la única persona de la disciplina que posee un noveno dan (máxima graduación en las artes marciales coreanas).

A partir del segundo dan es obligatorio realizar cortes de bambú con espadas (singeom) durante la evaluación. La centralidad de esta práctica durante la ceremonia se acentúa separándola temporalmente del resto del examen por medio de una exhibición. Los maestros encargados de la demostración van rotando de examen a examen. Este año nuestra escuela es la encargada, y Jang, Go y Park han hecho sus deberes con seriedad. Vestidos con trajes de entrenamiento (dobok) azules, acomodan el escenario para el espectáculo. La demostración deslumbra a los novatos, que perciben el abismo técnico que los separa de los maestros. Mientras Go, a un costado, muestra el poder de la velocidad apagando varias velas con el roce de la espada en un corte horizontal, Jang y Park se mueven velozmente girando, saltando y cortando bambús colocados en distintas posiciones y separados por varios metros de distancia. De fondo repiquetea una música de ritmo acelerado con notas altisonantes que produce emoción en los espectadores. A cada corte le siguen expresiones colectivas de admiración, mientras seguimos alucinados el despliegue de habilidad.

Los movimientos se aceleran acompañados por el ritmo in crescendo de la música. Jang realiza una combinación de cinco cortes en un bambú colocado de forma horizontal sobre la punta de otro bambú, sin que se caiga. El estadio entero rompe en aplausos. Para finalizar, Go apaga más de una docena de velas al mismo tiempo con un solo corte, mientras Jang y Park cortan el mismo bambú intercalando sus movimientos. Los filos de las espadas pasan a centímetros de sus manos a medida que seccionan el tallo hasta reducirlo a unos diez centímetros. La audiencia continúa aplaudiendo y despide a los maestros en medio de chiflidos.

Casi dos horas después de iniciada la ceremonia nos disponemos a ver los cortes de bambú, que marcan el final de la evaluación. Según Jang, allí se produce el mayor porcentaje de fallos por examen. Por eso, se le permite a cada practicante dos oportunidades para ejecutar la secuencia. La cantidad de cortes equivalen al cinturón al que se aspira (dos para segundo dan, tres para tercero, etc.). Todo contribuye a volverlo el momento decisivo y el que mayor ansiedad genera en los entrenamientos. De los doce practicantes que se sometieron a la prueba en esta ocasión, solo dos consiguieron aprobar.

Hwang y Kim, los alumnos estrella de nuestra academia, tomaron el examen para cuarto dan ese día y ambos fallaron en esta instancia. En el último corte de la secuencia de Kim, el fragmento de bambú quedó colgando por una fina astilla. No obstante, recibió una ovación de aplausos de la tribuna y de los demás alumnos por la velocidad y fluidez de sus movimientos. "Aunque el corte es técnicamente incorrecto -me dice Go, cuando terminamos de aplaudir con fuerza-, quizá los evaluadores lo aprueben por la reacción del público". Lamentablemente no fue así, y nuestros ánimos caen por el piso. El fracaso se siente tanto en el grupo como en el individuo. La escuela de Jang tendrá que esperar otro año para contar con dos nuevos instructores. Kim y Hwang, por su parte, deberán postergar la posibilidad de conseguir empleo en el oficio. En el caso de Kim el golpe se siente más, porque es un obrero sub-empleado que conduce un camión para su padre, cuando hay demanda, y complementa su ingreso enseñando esgrima japonesa dos veces por semana en un instituto terciario de Geoje-do. La ilusión de emplearse a tiempo completo como instructor de gumdo se vislumbra desde hace meses en su horizonte, cuando comenzó a ahorrar para cubrir el elevado costo de la matrícula (450 dólares en 2008). Para ello redobló su dedicación en los entrenamientos, interrumpiendo la carrera de electricista que cursa en una academia técnica de Gohyeon, su pueblo natal. 
Cabizbajos, nuestros compañeros se unen al grupo mientras unos maestros retiran los restos de bambú dispersos por el estadio y los armatostes usados como soporte. La voz del locutor a través del micrófono nos llama a formar. Volvemos a nuestras posiciones y saludamos a los maestros e instructores que presidieron el ritual. El resto es un poco caótico. La mayoría de los pupilos rompen filas, sonrientes, y se reúnen con familiares y compañeros de práctica para tomar fotografías grupales o imitando concienzudas posiciones marciales.

De regreso, intentamos disminuir el sentimiento de angustia que embarga a nuestros colegas más versados. Go y yo comentamos enérgicamente la actitud intransigente de los evaluadores, mientras que Jang articula una explicación más convincente sobre el notable verdor de las cañas de bambú, que de lo contrario no se abrían astillado de esa manera. El próximo año, promete, supervisará personalmente la selección de las plantas. Pero Kim se desentiende del asunto centrando su atención en mi examen. Cariñosamente, apoya su mano en mi hombro y con una sonrisa me dice, "Ije nae dongsaeng-ibnida" (Ahora eres mi hermano menor)". (Notas de campo, Geoje-do, noviembre de 2008).

Luego de desunir simbólicamente al sujeto de la estructura organizacional mediante su separación temporal y espacial dentro del dojang, la transición se completa con una fase de reincorporación. A partir de ahora, el sujeto ritual

[S]e halla de nuevo en un estado relativamente estable y, en virtud de ello, tiene derechos y obligaciones vis a vis otros de un tipo claramente definido y 'estructural'. De él se espera que se comporte de acuerdo con ciertas normas dictadas por la costumbre y ciertos principios éticos vinculantes para quienes ocupan posiciones sociales en un sistema de tales posiciones (Turner, 1988, p. 102).

Esta fase constituye una vuelta a la normalidad, con la salvedad que ahora el practicante posee un capital cultural incorporado e institucionalizado que lo acompañará el resto de su vida y que permea sus modalidades de interacción y estrategias en la cotidianeidad de la vida marcial. En su nuevo estado el iniciado puede involucrarse en situaciones que le estaban vedadas en tanto neófito -como la posibilidad de comprar una espada real en gumdo-, y que contribuyen a objetivar los atributos recientemente adquiridos.

Las características del mercado de insumos marciales y las personas que circulan por él constituyen un conocimiento práctico que se adquiere, al igual que los aspectos más esotéricos del entrenamiento, mediante una inmersión activa e intuitiva en las esferas de producción y consumo de los bienes del arte. Para realizar buenas transacciones hace falta conocer la idiosincrasia del grupo, por lo que generalmente el maestro asiste a su alumno en la adquisición del sable. También se encarga de acompañarlo a inscribir el arma en la policía, donde da su palabra de que el propietario es mayor de edad y poseedor de un cinturón negro (esto es necesario porque el trámite de entrega de los títulos por la Asociación suele demorar entre dos y tres meses). El proceso de agregación se completa con un convite del iniciado a participar en una práctica exclusiva para veteranos: la sesión de cortes de bambú. Esta actividad se organiza en días de no-entrenamiento y usualmente al aire libre, no solo por el espacio que demanda sino sobre todo porque es una puesta en escena de las habilidades del grupo para el público no iniciado.

b) "El domingo por la mañana Go y Park me visitan en el gimnasio de Jang. Al llegar, Go chequea rápidamente que Jang no esté presente. Acto seguido se echa a sus anchas en el sofá favorito de su maestro y lo llama por celular. "Está compitiendo en un torneo de arquería en un pueblo cercano. Termina después del mediodía", nos informa. Mientras tanto, se dedica a organizar la salida. Contacta a Lee, Kim, Hwang y otros dos cinturones negros que actualmente gozan de licencia del servicio militar obligatorio, y nos juntamos en el gym a esperar al maestro. Cuando el equipo finalmente está reunido nos dividimos en dos vehículos para recolectar los bambús. A causa de una tormenta la noche anterior los caminos están empantanados y no podemos acceder a la base de la montaña que suelen visitar para tal fin. La antigüedad de la planta es importante para una buena práctica. Si tiene más de dos años, el tallo es demasiado grueso y seco. Por el contrario, si es muy joven el verdor hace que se astille. Por eso hay que rotar los lugares de recolección. 
Jang nos dirige a una zona que parece conocer bien. Estacionamos entre unos árboles ocultando intencionalmente los coches, y nos adentramos en la mata de bambús de más de diez metros de alto. A poco de perdernos tras el follaje nos recibe una nube de mosquitos, de los que nos defendemos con repelente. Las arañas verdes y rojas, sin embargo, son otro tema, y hay que esquivar con cuidado sus extensas redes. Armados con serruchos comenzamos nuestra recolección, cortando primero la base de los bambús, por arriba del primer tallo, y luego separando la planta en partes de seis tallos cada una (altura adecuada para entrenar cortes). Luego de recolectar unos veinte o veinticinco bambús de aproximadamente cuatro metros, que equivalen a unas cien piezas, Jang considera que nuestro botín es suficiente y comenzamos a asegurar los fajos. Mientras los cargamos en las camionetas veo que un vecino se acerca cauteloso. Jang también lo nota y salta del vehículo para interceptarlo, saludando con respeto. Comienzan un intercambio verbal acelerado y en cinco minutos ya son cuatro los pobladores que rodean al maestro, todos hablando al mismo tiempo. Una señora mayor y encorvada irrumpe visiblemente alterada, agitando su mano en el aire. Aunque no puedo escuchar todo lo que dicen, es claro que reclaman la pertenencia del terreno y, por extensión, de las plantas. La señora se mueve inquieta mientras un hombre de mediana edad, posiblemente su hijo, intenta alejarla. Go, despreocupado, nos hace señas de que sigamos cargando, lo que enoja más a la mujer. Terminamos de asegurar los fardos y huimos amuchados en un vehículo, dejando a Go y Jang con los vecinos del poblado.

Estacionamos en una plaza amplia construida por la empresa Daewoo, propietaria del segundo astillero más grande de Corea del Sur, en la cual los obreros suelen recrearse con sus familias o amigos durante los días de descanso. Es fácil identificarlos por el mameluco gris de la compañía, que visten aún un domingo por la tarde. Preparamos las máquinas que sostienen los bambús para comenzar el entrenamiento. Jang y Go se nos unen cuando terminamos la preparación. Park arranca la sesión con unos cortes para entrar en calor. De paso, me enseña la secuencia que se evalúa durante el examen para segundo dan, y me hace lugar para que la ensaye. De entrada siento que lo hago bastante bien, pero Jang me indica que separe más los pies para ajustar la posición. De a poco me voy acostumbrando al movimiento descendente de derecha a izquierda. Los cortes salen con naturalidad. Cuando pruebo la secuencia completa no consigo completar el segundo movimiento y mi espada se atora en la caña, provocando la risa de todos. "Si fuera un enemigo, ya estarías muerto'“', bromea Park.

Media hora después, Jang recibe una llamada y le pide a Hwang que lo acompañe. Entonces me percato que Go tampoco está presente. Continúo entrenando sin darle mayor importancia. Casi una hora y media más tarde vuelve Go, y con gesto cansado nos informa que tuvieron que reunirse con los vecinos porque demandaban una compensación dineraria por las plantas que hurtamos. Le pregunto por qué los vecinos se habían molestado tanto. "En Corea -me responde- algunos lugares son espirituales, y ciertas familias son encargadas de protegerlos. Bueno, aquel era uno de esos lugares". Es común en los poblados más pequeños y tradicionales que las familias extensas sean propietarias de una pequeña montaña donde entierran a sus muertos. El varón de mayor edad en la familia es el encargado de velar por la seguridad del lugar. De allí el visible enojo de la señora, no solo por la transgresión de nuestro grupo sino también por la pasividad que mostró su hijo ante la afrenta. Finalmente, Jang regresa con la noticia de que no tuvo que pagar más que lo que Go llama "lips service". Es decir, alabar la generosidad de los vecinos por cedernos los frutos de un terreno tan preciado para su familia, garantizando que iban a ser usados rigurosamente en un entrenamiento de artes marciales. Una explicación que consigue apaciguarlos notablemente". (Notas de campo. Geoje-do, abril de 2009).

La infracción provocada por nuestro grupo activa la confrontación entre dos regímenes morales íntimamente relacionados, en una disputa por definir cuál tiene mayor jerarquía. Por un lado está en juego la importancia otorgada a la propiedad privada y a las creencias ancestrales. Por el otro, el estatus atribuido a las disciplinas marciales. La disputa patrimonial se replica en el plano simbólico en relación al honor, indicando un "nexo entre los ideales de una sociedad y su reproducción en el individuo, a través de su aspiración a personificarlos" (Pitt-Rivers, 1966, p. 22, traducción propia).

En esta confrontación, los contendientes movilizan repertorios discursivos que resultan razonables para el otro grupo. En última instancia, el valor social conferido a las artes marciales, 
hecho cuerpo en la reconocida figura de Jang, se impone a la transgresión sobre la propiedad privada y las creencias religiosas. En esta disputa se activa una función central del honor, que es la de simultáneamente igualar y jerarquizar a los sujetos en la interacción (Pitt-Rivers, 1966). La concesión que otorga el grupo vulnerado a los transgresores, es facilitada por la disposición del maestro de gumdo a humillarse frente a personas que gozan de menor jerarquía social que él. Al reconocerles su generosidad resguarda el honor familiar, que de esta manera no resulta mancillado. La familia puede ceder sabiendo que ha contribuido a una práctica socialmente respetable. Y además ha sido reconocida por ello. La estrategia de Jang es particularmente efectiva porque permite al primogénito y cabeza de la familia, presumiblemente el más afectado por la interacción, resguardar su honor ante la opinión pública y su grupo de pertenencia. A su reclamo se le ha concedido reputación (Pitt-Rivers, 1966). Y no cualquier reputación sino una de peso. De esta manera, se ha salvado del ridículo.

\section{De "hijo de la madre" a "hijo del padre"}

Analizar las relaciones de género en las artes marciales coreanas supone tomar en serio la propuesta bourdesiana de estudiar los habitus ${ }^{10}$ deportivos/marciales buscando desentrañar en la práctica las relaciones de homología que este campo social mantiene con otros campos y clases sociales, en el universo de los posibles culturales (Bourdieu, 1978, 2007b). Antes, hemos señalado que en la sociedad coreana la madre constituye una figura central en la crianza del niño. El principio confucionista de división sexual del trabajo, instaurado como ideología estatal durante la dinastía Joseon (1392-1897 DC), encuentra continuidad en la vida moderna (Yang \& Rosenblat, 2001; Seth, 2008). Recae sobre las madres la responsabilidad de elegir las academias de sus hijos y atender a sus necesidades durante la educación formal, que comienza a una edad cada vez más temprana (Seth, 2008; Millán, 2015). También son las encargadas de producir y cultivar lazos sociales duraderos y capitalizables para éstos en el plano educativo. De allí que algunos maestros e instructores suelan describirlas como "apéndice de los estudiantes".

Hemos mostrado que la ceremonia de graduación en artes marciales colabora en la socialización del estudiante coreano, internalizando categorías que le permiten establecer relaciones que funcionan en varias esferas sociales, y le permiten elaborar estrategias de acción e interacción en la sociedad de la que es miembro. El análisis de este ritual de consagración debe atender también a otra función social igualmente importante: la de "separar a aquellos que lo han experimentado, no de los que no lo han experimentado todavía, sino de aquellos que no lo experimentarán de ninguna manera, y el de instituir, así, una diferencia duradera entre aquellos a los que atañe este rito y a los que no les atañe" (Bourdieu, 1993, p. 113).

La asociación recurrente entre el "espíritu" de los guerreros Hwarang de la dinastía Silla, el "espíritu nacional" del pueblo coreano, y el "espíritu indomable" que infunden las disciplinas marciales (Forrest \& Forrest-Blincoe, 2018), indica que éstas, al igual que la política, la economía o la guerra, son percibidas como ocupaciones masculinas, constituidas en torno a valores como la autoridad y el vigor (Lee, Gibson \& Yi, 2013). En este sentido, "el efecto más importante del rito es el que pasa más desapercibido: al tratar de manera distinta a los hombres y a las mujeres, el rito consagra la diferencia, la instituye" (Bourdieu, 1993, p. 113) Mediante la persuasión de una pedagogía implícita, el acto de consagración de obtener un cinturón negro en la cultura coreana inculca los principios arbitrarios de un orden social androcéntrico.

10 Entendidos como "sistemas de disposiciones duraderas y transferibles, estructuras estructuradas predispuestas a funcionar como estructuras estructurantes, es decir, como principios generadores y organizadores de prácticas y de representaciones" (Bourdieu, 2007, p. 86), que son producto de la exposición duradera a determinadas condiciones de existencia. Estas disposiciones son objetivamente compatibles con aquellas condiciones, sin ser el resultado del cálculo racional o de la adaptación mecánica a leyes, sino por la relación de homología entre condiciones externas -objetividad hecha cosas-y esquemas internos -objetividad hecha carne-. De esta manera, "las disposiciones interiores, interiorización de la exterioridad, permiten a las fuerzas exteriores ejercerse, pero según la lógica específica de los organismos en los cuales están incorporadas, es decir de manera duradera, sistemáticamente y no mecánica" (p. 89). 
Al seguir las trayectorias de mujeres en la jerarquía marcial, observamos una creciente deserción hasta llegar a su casi virtual ausencia en los rangos de instructor y de maestro, y su completa desaparición en los puestos directivos. Comentando esta observación con Go, me confiesa que

Hay algunas mujeres, pero la mayoría deja de entrenar antes de llegar a cinturón negro. En gumdo hay muy pocas Kwanjang-nim (Maestras). No está muy bien visto que una mujer enseñe artes marciales. Tiene que ver con las viejas tradiciones coreanas. Antes, las mujeres se quedaban en casa. De la casa de sus padres se mudaban a la de su esposo. Ahora eso está cambiando, pero no tan rápido (Fragmento de entrevista. Geoje-do, septiembre de 2008).

La ausencia de mecanismos institucionales para eliminar a las practicantes incluso asume la forma de principio ético, como rechazo explícito a las distinciones de sexo, etnia, religión o clase social. En la práctica, sin embargo, las normas que orientan las relaciones entre géneros no reflejan este discurso. La percepción acerca de los sexos y de lo que le corresponde a cada uno en el orden de las cosas, instituye de esta manera formas objetivas de restricción que operan eficazmente, puesto que son disimuladas por una toma consciente de posición en sentido contrario.

De esta manera, las organizaciones de artes marciales limitan sistemáticamente el progreso femenino en el oficio, resguardando su práctica más avanzada -por ende, más distinguida- para los varones. Esta deserción suele ser explicada por las madres que deciden retirar a sus hijas del dojang, como una inadecuación entre las características asociadas a lo femenino y el tipo de actividad que la práctica marcial requiere, mediante afirmaciones como: "esto no es para ella", "le resulta muy exigente" o "no puede seguir el ritmo de sus compañeros".

En condiciones análogas este fenómeno se replica en otros campos sociales, como la participación femenina en el mercado laboral (Lee M.-J., 2008) o en las esferas políticas (Eun, 2008). La creciente cantidad de mujeres jóvenes de Geoje-do que se incorporan diariamente como empleadas en los astilleros de Daewoo y Samsung, lo hacen mayormente en puestos de atención al público y secretariado. Ocupaciones percibidas como feminizadas puesto que suponen dedicaciones parciales y de menor esfuerzo físico. En Corea del Sur, las mujeres empleadas de forma permanente alcanzan apenas el $25,6 \%$ del total de trabajadoras (Lee, 2008). Cuando Jang explica el carácter temporal de estas ocupaciones laborales, lo hace en función de la expectativa social de que "una vez que formen familia, renuncien a sus trabajos"11. La percepción de este maestro de gumdo no difiere en demasía del sentido común de gran parte de los coreanos. Así, "el principio de 'separación del hombre y la esposa' que establecía distintas esferas de actividad para hombres y mujeres casados se transfirió de forma natural en la familia como clara división de los roles de género. Esta fue la norma más fundamental en la doctrina confuciana que prevaleció como la ideología nacional." (Eun, 2008, p. 148, traducción propia del original, en inglés). En efecto, "la toma de conciencia de la identidad sexual y la incorporación de las disposiciones asociadas a una definición social determinada de las funciones sociales que incumben a los hombres y a las mujeres van a la par con la adopción de una visión socialmente definida de la división sexual del trabajo" (Bourdieu, 2007a, p. 127).

De esta manera, la experiencia del proceso ritual como una transformación ontológica adopta características sociales diferenciales para cada sexo. Los practicantes de artes marciales varones experimentan la conversión en cinturón negro en simultáneo con su conversión en adultos y trabajadores. Contrariamente, las mujeres lo viven como renuncia a la posibilidad de alcanzar este estatus, mientras son normativamente disuadidas a aceptar que su lugar en la sociedad está en el hogar, junto a su esposo y sus hijos.

\footnotetext{
11 El peso de esta afirmación no solo se percibe en el plano discursivo, sino también en las prácticas de contratación y leyes laborales. Tan solo tres décadas atrás, "se pedía a las mujeres que firmasen contratos que exigían su resignación una vez que se casasen.” (Lee M.-J., 2008, p. 177). Aunque la sociedad coreana ha experimentado drásticas transformaciones desde entonces, "con respecto a la desigualdad y la discriminación en las oportunidades de empleo, los salarios, la promoción, la protección legal de las madres y el acoso sexual y la violencia, las mujeres siguen viviendo en una posición relativamente desfavorecida." (Lee 0.-J., 2008, p. 174, traducciones propias del original, en inglés).
} 


\section{Conclusiones}

La consolidación y difusión de las artes marciales coreanas es el resultado de un proyecto nacionalista, que comienza a tomar forma luego de la devastación producida por la ocupación japonesa y la Guerra de Corea. En aquellas, los sucesivos gobiernos militares y democráticos han depositado un mandato de unificación nacional, encarnado en la enseñanza de valores considerados fundamentales para la sociedad. Este proyecto permitió reescribir de manera legítima la historia oficial de Corea del Sur, a partir de un criterio de autoctonía en el que se oscurecen o eliminan las influencias históricas de Japón y China sobre la cultura nativa.

Desde que ingresan en edad escolar, los varones coreanos están continuamente expuestos a la influencia de las artes marciales, sea por medio de la escuela, donde el taekwondo forma parte de los currículos básicos, en las academias privadas, durante el servicio militar obligatorio, o por los medios de comunicación. Estos sistemas de combate contribuyen de manera decisiva a producir una determinada forma de percibir el mundo y de relacionarse con otros miembros de la sociedad. Los principios morales que inculcan las disciplinas marciales coreanas son apropiados por los practicantes mediante ritos de paso, que tienen por sentido objetivar las jerarquías del mundo marcial. Al analizar el sistema de rangos en que se organiza su práctica y los rituales necesarios para moverse por este espacio simbólicamente estructurado, hemos señalado su importancia para regular las interacciones entre practicantes, a partir de instituir jerarquías y valores como el respeto a la autoridad y la importancia de pertenecer a un colectivo. Su análisis también revela que, en su funcionamiento regular, las organizaciones de artes marciales coreanas regulan las relaciones entre sexos, promoviendo o desalentando el progreso de los neófitos en el oficio según su género. En el caso de las mujeres, el avance por el sistema de rangos marciales va siendo progresivamente limitado, consagrando su práctica más avanzada, por ende, distinguida, a los hombres. De esta forma contribuyen a la reproducción de una forma específica de dominación masculina, que encuentra homología en otros campos sociales de la sociedad coreana, como la esfera doméstica, el mercado laboral o la carrera política. Es allí donde se visualiza la principal contradicción entre organizaciones que se piensan inclusivas, y las desigualdades que contribuyen a reproducir en la práctica.

Asumir esta divergencia es el primer paso para romper con el sentido común que la sustenta; proceso que ya ha mostrado importantes resultados en relación a la educación universitaria (Eun, 2008; Lee J.-K., 2008; Lee 0.-J., 2008). Resta conocer las implicancias que este cambio podría generar en la enseñanza y aprendizaje de oficios en los cuales el culto a la masculinidad tiene un papel constitutivo. Lo que sí podemos afirmar es que, aunque incipiente, no es una preocupación ajena a buena parte de la sociedad coreana. Sobre todo entre los más jóvenes, quienes ya están poniendo en marcha procesos de crítica y transformación de las desigualdades de género en diversos espacios de la vida social.

\section{Financiación}

Esta investigación contó con el financiamiento otorgado por el CONICET mediante una beca doctoral. También agradezco la ayuda económica que me brindó la Academy of Korean Studies mediante un premio a jóvenes investigadores para el estudio de la cultura coreana.

\section{Agradecimientos}

Mi trabajo no hubiera sido posible sin el apoyo incondicional de maestros y amigos en Corea del Sur. Asimismo, agradezco los valiosos comentarios de Gabriel Noel, Denis Baranger, Yamila Irupé Nuñez, Antonio Millán y Elías Gómez. Todos ellos están, por supuesto, autorizados a la habitual absolución de la culpa sobre cualquier omisión que haya sobrevivido a su escrutinio. Por último, me gustaría agradecer a los revisores del presente artículo sus valiosas observaciones, que sin duda han permitido mejorar su calidad final.

\section{Referencias}

Balbi, F. (2017). Moral e interés. Una perspectiva antropológica. Publicar, 23, 9-30. 
Bourdieu, P. (1978). Sport and Social Class. Social Science Information, 17(6), 819-840. doi: $10.1177 / 053901847801700603$

Bourdieu, P. (1993). Los ritos como actos de institución. En J. Pitt-Rivers \& J. G. Peristiany (Eds.), Honor y gracia (pp. 111-123). Madrid: Alianza Universidad

Bourdieu, P. (2007a). El sentido práctico. Buenos Aires: Siglo XXI Editores.

Bourdieu, P. (2007b). Programa para una sociología del deporte. En Cosas Dichas (pp. 173-184). Barcelona: Gedisa.

Bowman, P. (2016). Making Martial Arts History Matter. The International Journal of the History of Sport, 33(9), 915-933. doi: 10.1080/09523367.2016.1212842

Channon, A. \& Jennings, G. (2014). Exploring Embodiment through Martial Arts and Combat Sports: A Review of Empirical Research. Sport in Society, 17(6), 773-789. Doi $\underline{10.1080 / 17430437.2014 .882906}$

Choi, H. H. (1996). Taekwondo (el arte coreano de la defensa personal). Buenos Aires: ITF.

Crossley, N. (2005). Mapping Reflexive Body Techniques: On Body Modification and Maintenance. Body \& Society, 11(1), 1-35. doi: $10.1177 / 1357034 X 05049848$

Elias, N. (2016). Introducción: Ensayo teórico sobre las relaciones entre establecidos y marginados. En N. Elias \& J. Scotson, Establecidos y marginados. Una investigación sociológica sobre problemas comunitarios (pp. 27-71). México: Fondo de Cultura Económica.

Eun, K.-S. (2008). Family values changing - but still conservative. En K.-D. Kim (Ed.), Social change in Korea (pp. 146-156). Paju: The Korea Herald.

Evans-Pritchard, E. E. (1992). Los Nuer. Barcelona: Anagrama.

Forrest, J. \& Forrest-Blincoe, B. (2018). Kimchi, K-Pop and Taekwondo: The Nationalization of South Korean Martial Arts. Ido Movement for Culture. Journal of Martial Arts Anthropology, 18(2), 114. doi: $10.14589 /$ ido.18.2.1

Graham, E. (2013). 'There Is No Try in Tae Kwon Do': Reflexive Body Techniques in Action. En R. Sánchez García \& D. Spencer (Eds.), Fighting Scholars. Habitus and Ethnographies of Martial Arts and Combat Sports (pp. 63-77). London \& New York: Anthem Press.

Howell, S. (2005). Introduction. En S. Howell (Ed.), The Ethnography of Moralities (pp. 1-21). New York: Routledge.

Hwang, K. (2010). A History of Korea. New York: Palgrave Macmillan.

Kim, Y.-G. (2012). 15 códigos de la cultura coreana. Buenos Aires: Bajo La Luna.

Lee, M.-J. (2008). Dual earners call for family-friendly society. En K.-D. Kim (Ed.), Social change in Korea (pp. 175-183). Paju: The Korea Herald.

Lee, O.-J. (2008). More gender equality, but women still held back. En K.-D. Kim (Ed.), Social change in Korea (pp. 167-174). Paju: The Korea Herald.

Lee, J.-D., Gilson, T. \& Yi, B. (2013). Cultural Explications in Martial Arts, Taekwondo: Collectivistic, Power-centered, Probabilistic and Masculine. Taekwondo Journal of Kukkiwon, 4(1), 1-30.

Mauss, M. (1979). Ensayo sobre los dones. Motivo y forma del cambio en las sociedades primitivas. En Sociología y Antropología (pp. 153-263). Madrid: TECNOS.

Millán, G. (2015). El Dojang: escuela de disciplina y moralidad. Revista de Artes Marciales Asiáticas, 10(1), 1-15. doi: $10.18002 /$ rama.v10i1.1445

Moenig, U. (2017). Taekwondo: From a Martial Art to a Martial Sport. New York: Routledge. doi: $\underline{10.4324 / 9781315733227}$

Moenig, U. \& Kim, M. (2016). The Invention of Taekwondo Tradition, 1945-1972: When Mythology Becomes 'History'. Acta Koreana, 19(2), 131-164.

Na, E.-Y. (2008). Collectivism vs. individualism. En K.-D. Kim (Ed.), Social change in Korea (pp. 118126). Paju: The Korea Herald.

Noel, G. (2014). Presentación. Las dimensiones morales de la vida colectiva. Exploraciones desde los estudios sociales de las moralidades. Papeles de trabajo, 8(13), 14-32.

Nye, J. \& Kim, Y. (2013). Soft Power and the Korean Wave. En Y. Kim (Ed.), The Korean Wave: Korean Media Go Global (pp. 31-42). New York: Routledge.

Park, G.-S. (2008). Korean society caught in post-authoritarianism trap. En K.-D. Kim (Ed.), Social change in Korea (pp. 127-135). Paju: The Korea Herald.

Passeron, J. C. (2011). Nombres y Trabajos. En El razonamiento sociológico. El espacio comparativo de las pruebas históricas. Madrid: Siglo XXI. 
Peristiany, J. G. (1966). Introduction. En J. G. Peristiany (Ed.), Honour and Shame. The Values of Mediterranean Society (pp. 9-18). Chicago: University of Chicago Press.

Peristiany, J. G. \& Pitt-Rivers, J. (2005). Introduction. En J. G. Peristiany \& J. Pitt-Rivers (Eds.), Honour and Grace in Anthropology (pp. 1-17). Cambridge: Cambridge University Press.

Pitt-Rivers, J. (1966). Honor and Social Status. En J. G. Peristiany (Ed.), Honor and Shame. The Values of Mediterranean Society (pp. 20-77). Chicago: University of Chicago Press.

Sánchez García, R. (2008). Análisis etnometodológico sobre el dinamismo del habitus en Bourdieu y Elias dentro del desarrollo de actividades corporales. Reis. Revista Española de Investigaciones Sociológicas, 124, 209-231.

Sánchez García, R. \& Spencer, D., Eds. (2013). Fighting Scholars. Habitus and Ethnographies of Martial Arts and Combat Sports. London \& New York: Anthem Press. doi: $10.2307 / \mathrm{j} . \mathrm{ctt} 1 \mathrm{gxpddq}$

Seth, M. J. (2008). Fiebre educativa: sociedad, política, y el anhelo de conocimiento en Corea del Sur. Buenos Aires: Prometeo.

Seth, M. J. (2016). A Concise History of Korea. From Antiquity to the Present. Maryland: Rowman \& Littlefield.

Simmel, G. (2002). El ámbito de la sociología. En Cuestiones fundamentales de sociología (pp. 23-55). Barcelona: Gedisa.

Turner, V. (1988). El proceso ritual. Estructura y antiestructura. Madrid: Taurus.

Wacquant, L. (2006). Entre las cuerdas. Cuadernos de un aprendiz de boxeador. Buenos Aires: Siglo XXI Editores.

Wacquant, L. (2012). Chicago Fade: volver a poner el cuerpo del investigador en juego. Astrolabio. Nueva Época, 9, 157-167.

Weber, M. (2014). Conceptos sociológicos fundamentales. En Economía y sociedad (pp. 97-128). México: Fondo de Cultura Económica.

Yang, J.-H. (2008). Changing values cause ideological confusion. En K.-D. Kim (Ed.), Social change in Korea (pp. 157-166). Paju: The Korea Herald.

Yang, S. \& Rosenblatt, P. C. (2001). Shame in Korean Families. Journal of Comparative Family Studies, 32(3), 361-375. doi: $10.3138 /$ jcfs.32.3.361

\section{Author's biographical data}

Gonzalo Arial Millán (Argentina). Licenciado en Antropología Social y Docente del Departamento de Antropología Social, UNaM. Becario doctoral del CONICET en el Programa de Posgrado en Antropología Social (PPAS-UNaM). A partir de una estadía prolongada en la península coreana entre 2008 y 2009 desarrollé mi investigación sobre artes marciales y cambios sociales en dos ciudades industriales de Corea del Sur. En ese tiempo dicté clases de taekwondo y tomé clases de haidong gumdo, gungdo y lengua coreana. Soy tercer dan de taekwon-do ITF y segundo dan de haidong gumdo. Mi producción versa sobre el proceso de socialización de alumnos en las artes marciales coreanas y las tensiones que ha ido sufriendo la transmisión de estas disciplinas con el ingreso de Corea en la modernidad avanzada. E-mail: gonzalo.ariel.millán@gmail.com 